\title{
Non-destructive Electromagnetic Wave Sensor for Hazardous Biological Materials
}

\author{
${ }^{*}$ Kah Hou Teng \\ Built Enviornment and Sustainable \\ Technology (BEST) Research Institute, \\ Liverpool John Moores University, \\ Liverpool \\ k.h.teng@ljmu.ac.uk \\ Andy Shaw \\ Built Enviornment and Sustaible \\ Technology (BEST) Reserch Institute, \\ Liverpool John Moores University, \\ Liverpool \\ a.shaw@1jmu.ac.uk
}

\author{
Ibijoke Idowu \\ Built Enviornment and Sustainable \\ Technology (BEST) Research Institute, \\ Liverpool John Moores University, \\ Liverpool \\ I.A.Idowu@ljmu.ac.uk
}

Magomed Muradov

Built Enviornment and Sustainable

Technology (BEST) Research Institute,

Liverpool John Moores University,

Liverpool

m.muradov@ljmu.ac.uk

\author{
Patryk Kot \\ Built Enviornment and Sustainable \\ Technology (BEST) Research Institute, \\ Liverpool John Moores University, \\ Liverpool \\ p.kot@1jmu.ac.uk
}

\begin{abstract}
A novel non-destructive electromagnetic wave (EM) sensor for rapid identification of biological material is presented in this paper. Biological treats could be defined as biological agents such as bacteria spores, viruses and toxins. Spores can disable or kill people, animals and crops. Therefore, it is important to identify the hazard in rapid and non-destructive manner to make a safer environment. In this research, a 2.45 GHz microwave resonator was used to detect the dipliconic acid (DPA), which is the bio-maker of bacillus spores. A promising results were obtained by detecting the DPA from $0.001 \mathrm{M}-0.3 \mathrm{M}$ concentration at frequency of $2.4 \mathrm{GHz}$, which are the fundamental mode (TM101) of the designed cavity. In addition, different species of bacillus spores was detected at frequency approximate at $2.36 \mathrm{GHz}$. The results concluded that electromagnetic wave sensors may have the potential for use as a non-destructive and real time sensor to detect bacillus spores. The EM principle could be extended to detect different hazardous biological materials by identify the "finger print" of specific biological materials on different surfaces.
\end{abstract}

Keywords-Biological material, dipliconic acid (DPA), EM sensor, microwave cavity, non-destructive testing, $2.45 \mathrm{GHz}$

\section{INTRODUCTION}

Significant outbreaks of disease are among the highest impact risks faced by any society, threatening lives and causing disruption to public services and the economy. The UK Biological Security Strategy (HM Government, 2018) recognizes the importance of intervening early to prevent biological threats from emerging, or from spreading once they emerge. Large-scale disease outbreaks in animals or plants can be equally significant in terms of economic, environmental and social impact. Alongside the continuous management of diseases endemic to the UK, there were 22 outbreaks of exotic notifiable animal diseases in the UK between August 2000 and December 2017. The estimated incurred costs for the Government ranging from $£ 300,000$ to more than $£ 3$ billion. In addition, the Homeland Security and Emergency Management Market (Security world market, 2018 ) is projected to grow from an estimated USD 557.14 billion in 2018 to USD 742.06 billion by 2023. The researchers found that increasing terrorist threats and biohazard attacks and occurrences of unpredictable natural disasters due to ever-changing climatic conditions worldwide are key factors driving the growth of the homeland security and emergency management market.

Over the last decade, rapid technological developments have been noticed in electronics and microprocessor technology. There is a wide range of instrumentation available for chemical/biological monitoring. However, the existing technologies mostly are analytical instruments which require highly skilled and trained workers and are costly to manufacture and maintain in a laboratory [1]. Examples include fluorescence spectroscopy [2], mass spectrometry [3] , gas chromatography and matrix-assisted laser desorption ionization (MALDI) [4]. A number of investigators have described biosensors capable of analysis of [5, 6]. However, commercially available devices are large and require mains power, regular maintenance and constant supply of reagents to operate. In addition, provided systems are complex to operate and require time for sample analysis.

The independent discoveries of the bacterial spore by Tyndall, Koch and Cohn in the last quarter of the $19^{\text {th }}$ century [7] marked the beginning of the movement of spore research from the environment into the laboratory. There are different bacterial spores however, this project focuses on Bacillus species (Bacillus globigii, Bacillus thuringiensis). Bacillius globigii and bacillus thuringiensis are spore- forming bacterial speceies as seen in Figure 1. Both are used as surrogates for analysis of the potential properties of Bacillus anthracis spores. Bacillus thuringiensis is also used as an insecticide on a large scale. Pyridine-2,6-dicarboxylic acid (dipicolinic acid, DPA) as seen in Figure 2 is a unique constituent of all endospores from Bacillus and Clostridium species, and represents a substantial amount of the dry weight in bacterial spores (5-14\%) [8] DPA is localized in the spore core, and chelated with divalent cations. It has never been detected in vegetative cells. DPA is thus often used to detect the presence of bacterial spores. Hence they are often referred to as biomarkers. 


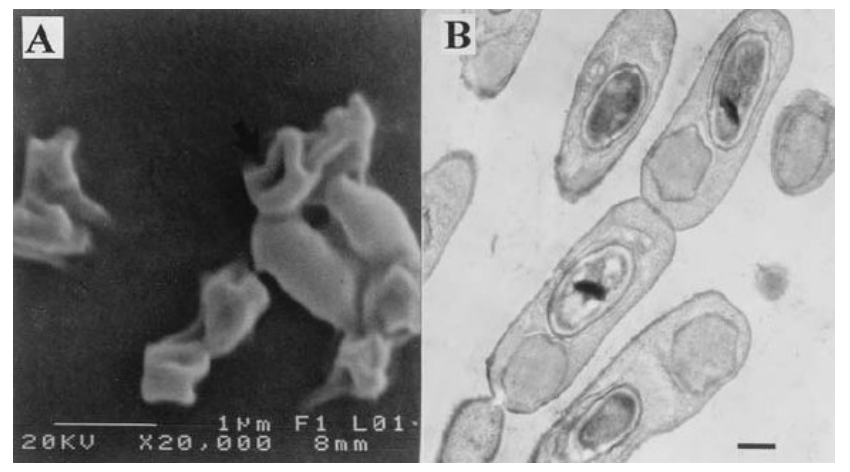

Figure 1: Scanned electron microphotograph of Bacillus thuringiensis [9].

(a)<smiles>O=C(O)c1ccccc1</smiles>

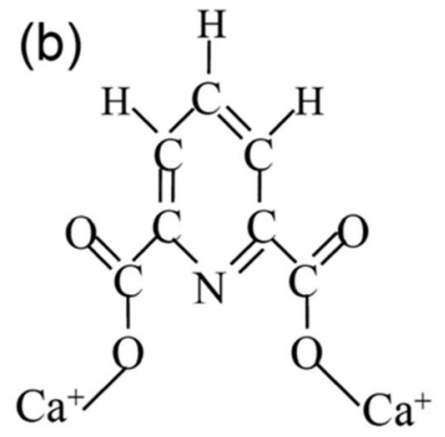

Figure 2. The molecular structure of (a) dipicolinic acid (DPA) and (b) calcium dipicolinic acid (CaDPA) [10].

This research aims to detect dipicolinc acid (DPA) using a $2.45 \mathrm{GHz}$ microwave cavity. The laboratory experimental work was conducted to prove the concept of electromagnetic wave capable to detect different concentration of DPA contains in a wet sampling. A good understanding of the characterization and unique signal spectrum of the DPA and varied concentration is expected using the wet-based sampling analysis. Therefore, the aim of this research is to develop a novel, non-destructive electromagnetic sensor for instant identification of hazardous biological material.

\section{EXPERIMENTAL PREPARATION AND METHODOLOGY}

\section{A. Microwave Theory and Application}

Materials can be studied from the data of their interactions with microwaves. This interaction can be realised in the form of a unique signal spectrum known as reflection coefficient (S11). Generally, these measurement quantities vary with the change in parameters such as permittivity and conductivity of the materials [11]. Permittivity is basically a measurement of the response of a dielectric medium to the applied microwaves that can be detected through its changing electric field. It depends on the material's ability to polarise in response to the applied field [12]. The two primary parameters that define permittivity are known as dielectric constant and dielectric loss of material [13]. (i) Dielectric constant ( $\left.\varepsilon^{\prime}\right)$ : The phenomena of energy storage and reduction in the wave velocity when EM waves pass through the material which is used to distinguish dielectric constant values of different materials. Different dielectric constant values are observed, because of the changes in polarisation inside the material.

(ii) Dielectric loss $\left(\varepsilon^{\prime \prime}\right)$ : The loss of electromagnetic energy propagating inside a dielectric material. The reduction of the wave magnitude is due to the rotation and oscillation of the molecules in response to the applied electric field and loss of energy owing to intermolecular interactions.

Changes in the materials' concentration, type, percentage etc. will be followed by the alteration of its permittivity yielding a unique signal spectrum when it interacts with microwave radiation. In this way, the material is characterised over the range of discrete frequencies

\section{B. $2.45 \mathrm{GHz}$ microwave cavity}

The designed cavity operates at a fundamental mode of operation $\left(\mathrm{TM}_{010}\right)$ at approximately $2.45 \mathrm{GHz}$. Equation (1) was used to calculate the resonant frequency for the fundamental mode in a cylindrical cavity.

$$
f_{n m l}=\frac{c}{2 \pi \sqrt{\mu_{r} \varepsilon_{r}}} \sqrt{\left(\frac{\rho_{m n}}{b}\right)^{2}-\left(\frac{l \pi}{d}\right)^{2}}
$$

Where $\mathrm{c}$ is the velocity of light, $\varepsilon_{r}$ is the relative permittivity of a material, $\mu_{r}$ is the relative permeability of a material, $\rho_{m n}$ is the mth root of the Besel function of the nth order, $b$ is the radius of the cavity and $d$ is the depth of the cavity.

Figure 3 shows the full dimension of the microwave cavity used in this research. The cylindrical cavity made of aluminum with the diameter of $9 \mathrm{~cm}$ and $10 \mathrm{~cm}$ height. On top of that, the sample was prepared in a sample tube of 15 $\mathrm{ml}$ with $3 \mathrm{~cm}$ diameter tube.

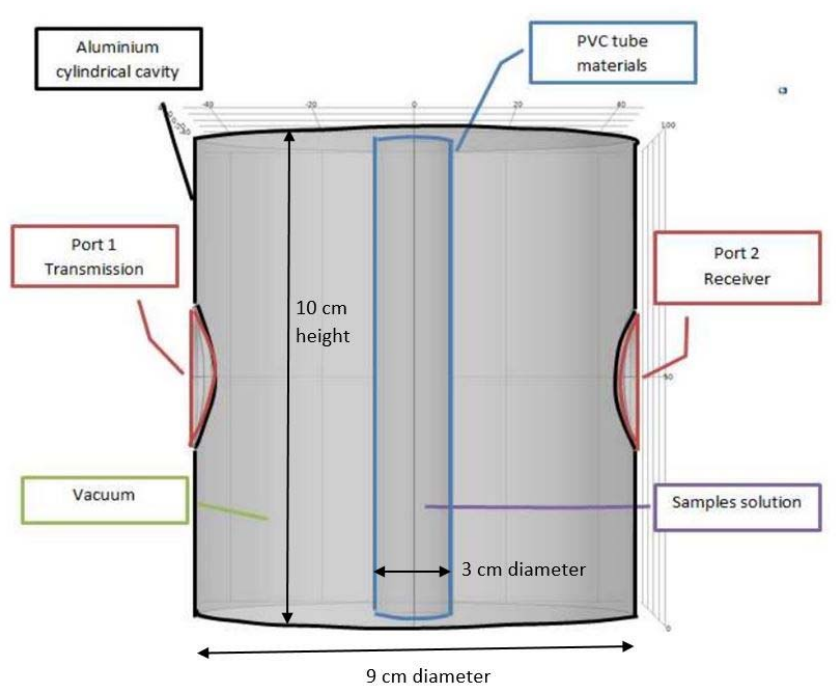

Figure 3. Full dimension of microwave cavity used for experiments. 


\section{Materials Preparation and Properties}

2, 6 pyridinedicarboxylic acid; DPA of assay purity $99 \%$, sodium hydroxide $(\mathrm{NaOH})$ of assay purity $\geqslant 98 \%$ and dimethyl sulfoxide(DMSO) of assay purity $99.7 \%$ were all purchased from Sigma- Aldrich UK while ethanol absolute HPLC grade with $99.99 \%$ purity, methanol analytical reagent grade with $99.99 \%$ purity, and acetone with $99 \%$ purity were purchased from Fisher Scientific. The deionized water was processed by water purification -Suez/purite Neptune ultimate catalogue number L300500. In order to prepare DPA solution, either $\mathrm{NaOH}$ or DMSO were used as solvents. Samples were prepared of concentration $0.001 \mathrm{M}-0.3 \mathrm{M}$.

\section{Experimental Setup}

In this investigation, the initial approach was taken to examine the responsive of electromagnetic wave (EMW) towards biological materials using a proven $2.45 \mathrm{GHz}$ microwave cavity resonator [14]. The microwave signal responsive were used to analyze the sample solution that occurs as function of DPA contents. Varied concentration of DPA $(0.001 \mathrm{M}-0.3 \mathrm{M})$ were prepared by dissolving DPA in $\mathrm{NaOH}$ and DMSO respectively. The samples were centrally positioned in the electric field of the microwave resonant cylindrical cavity Figure 4 . The reason the samples were measured at the center of cavity is because the distribution of the electric field magnitude was designed as shown in Figure 5 .

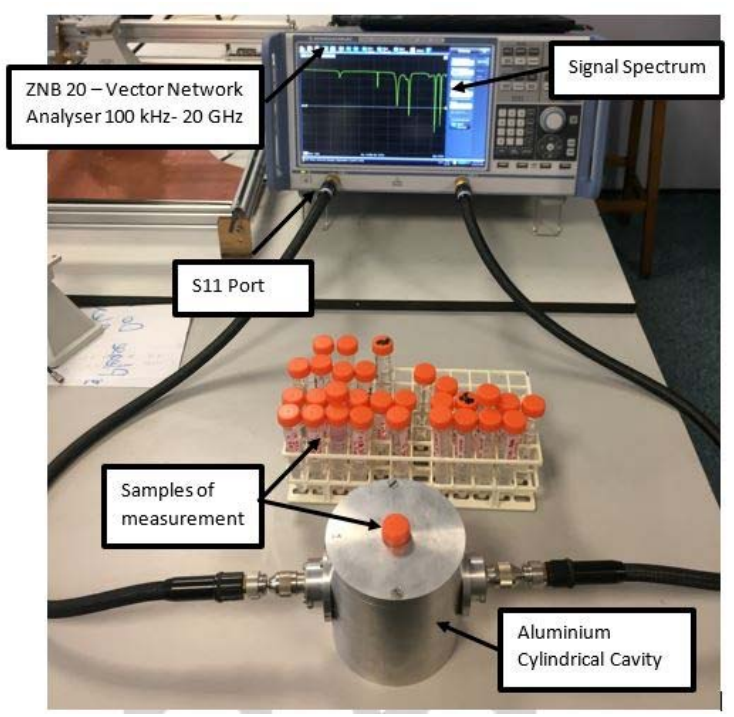

Figure 4. Experimental setup for microwave signal spectrum data collection on different samples

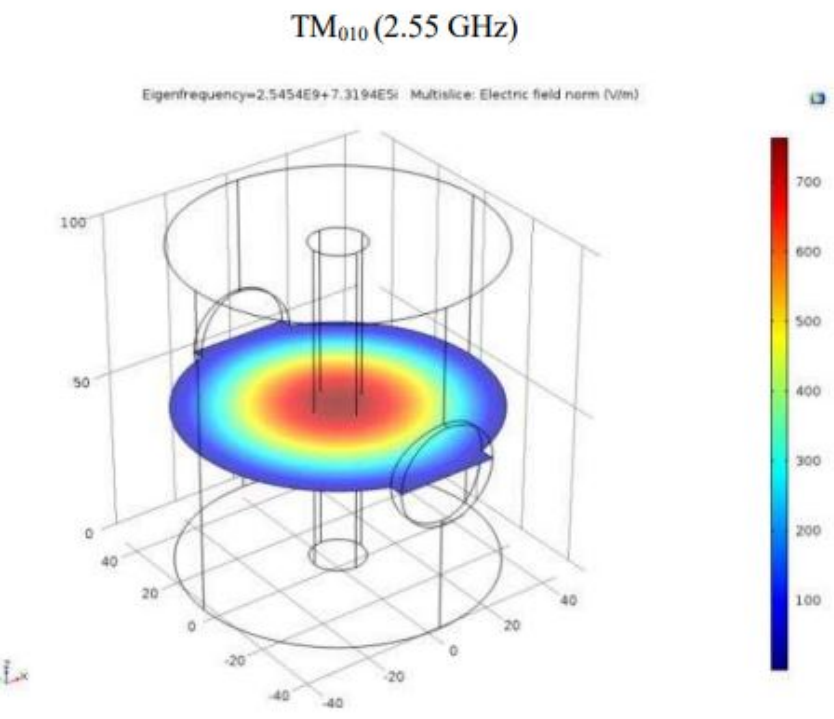

Figure 5. Fundamental mode of designed microwave cavity shows the strongest electric field magnitude in the center.

\section{E. Data Acquisition and Analysis}

Experiments were conducted to measure samples with different concentrations. All the microwave measurements were carried out as the reflected signal (S11). R\&S®ZNB 20 Vector Network Analyser, $100 \mathrm{kHz}-20 \mathrm{GHz}$ (Rohde \& Schwarz) was used to transmit the microwave signal and capture the microwave response. The measurements were recorded in the on board memory of the VNA. In the first approach of evaluation, the R\&S $\mathrm{R}$ NB (network analyser) were used for the research studies due to its powerful measurement capabilities for full sweep frequency range. A microwave input power of $1 \mathrm{~mW}(0 \mathrm{dBm})$ was launched into the cavity. Full two-port calibration was carried out to ensure the accuracy of the measurement. Feeding the microwave energy to the microwave sensor will be developed in future. This can be achieved by use of on board circuitry with and implemented machine learning algorithms. The EM sensor will be a hand held unit that will enable measurements over a wide area with a build in processing and instant display results.

To analyze the acquired samples data, perturbation theory of varied samples was used. Both the dielectric constant and dielectric loss of the material were characterized. On top of that, subtraction of the acquired samples from base solvent signature ( $\mathrm{NaOH}$ and $\mathrm{DMSO}$ ) based on magnitude and phase change were conducted to evaluate the area of interest for further analysis. Regression of the sample responsive to microwave signal were reported.

\section{RESULTS AND DICUSSION}

Reflected signal $\left(\mathrm{S}_{11}\right)$ measurements (real and imaginary) for DPA-NaOH and DPA-DMSO were obtained from the vector network analyser, Figures 5 and 6 show the scalar magnitude. 


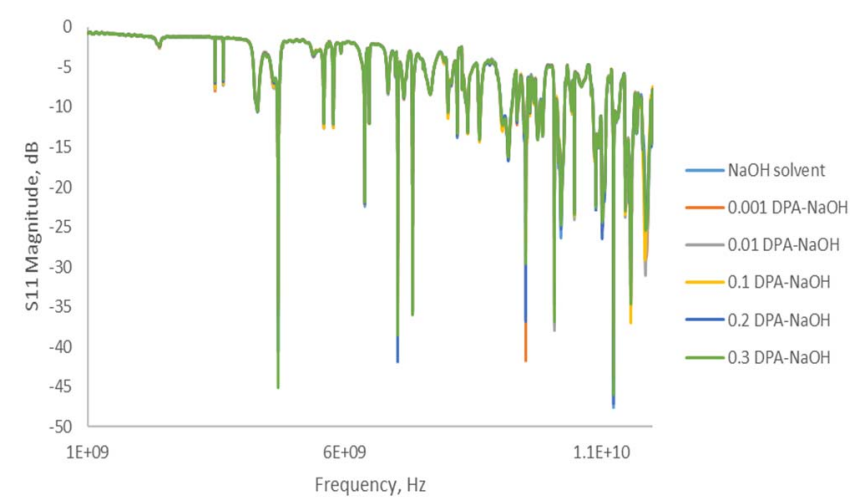

Figure $6 S_{11}$ raw data signal spectrum for DPA-NaOH from 1-12 GHz.

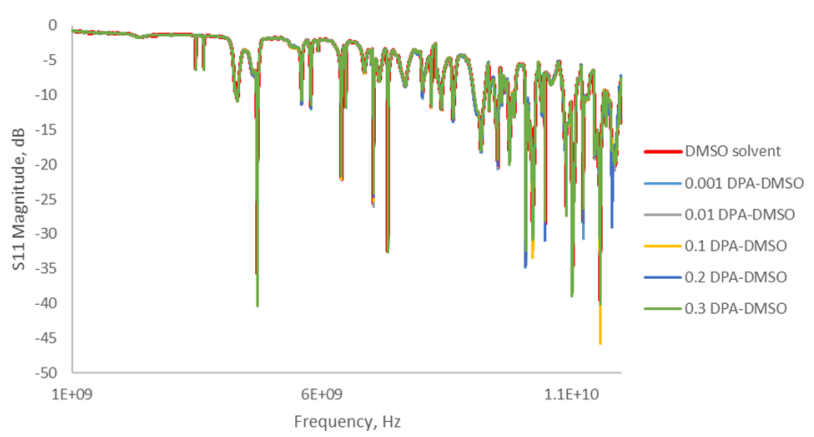

Figure $7 S_{11}$ raw data signal spectrum for DPA-DMSO from $1-12 \mathrm{GHz}$.

Following that, analysis of area of interest response frequency will be measured by deducted signal spectrum from based fluid using Equation 1.

$$
S_{11}=\sqrt{[\operatorname{Re}(\text { samp })-\operatorname{Re}(\text { base })]^{2}+[\operatorname{Im}(\text { samp })-\operatorname{Im}(\text { base })]^{2}}
$$

(1)

As seen in Figure 7 and Figure 8, varied concentrations of DPA were subtracted from base signature of $\mathrm{NaOH}$ and DMSO solvent to determine frequencies of interest for further evaluation to detect the biological material from $1-12 \mathrm{GHz}$ in 20000 data points.

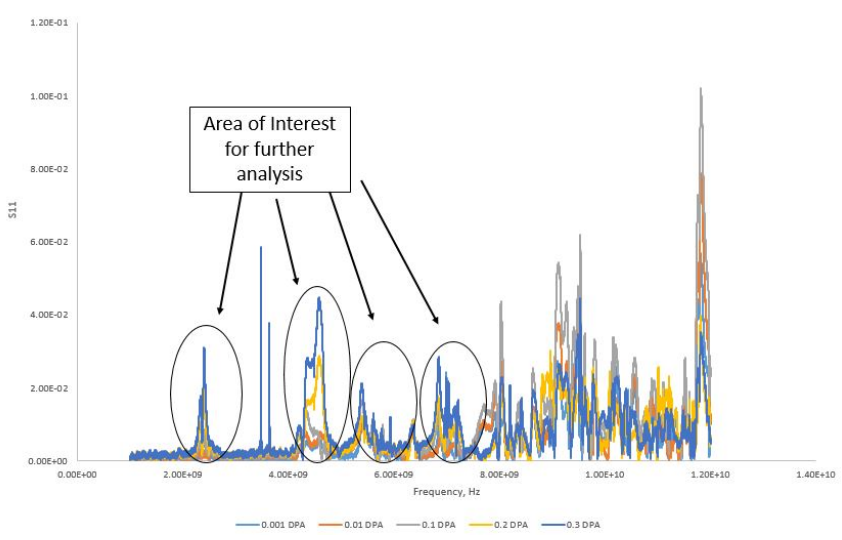

Figure 8. $S_{11}$ measurement of DPA subtract from based-fluid of $1 \mathrm{M} \mathrm{NaOH}$.

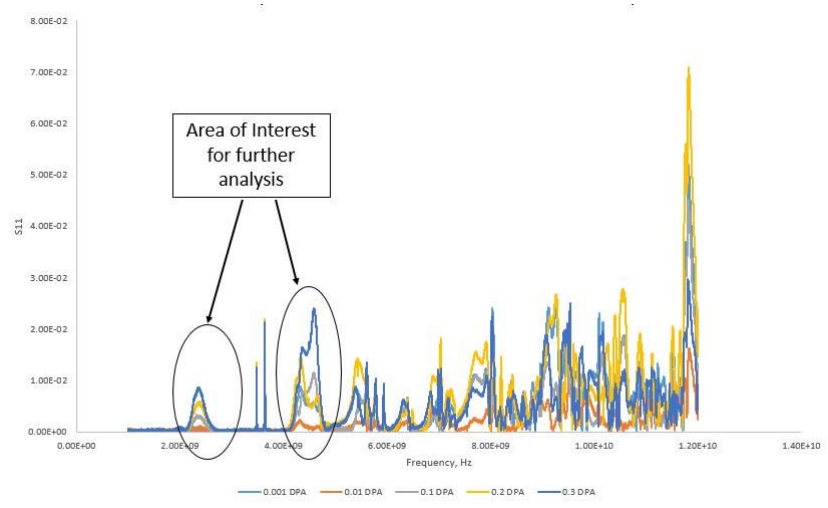

Figure 9. $S_{11}$ measurement of DPA subtract from based-fluid of DMSO

Further evaluation was conducted on frequencies of interest to verify the relationship of microwave signal responses to varied concentration of DPA. It is interesting to see that varied concentrations of DPA respond to microwaves in a linear trend. Figure 9 shows the varied concentration of DPA in $\mathrm{NaOH}$ responsive well to $\mathrm{S} 11$ at frequency $2.4 \mathrm{GHz}$ and $4.58 \mathrm{GHZ}$ with a regression of $\mathrm{R}^{2}=0.9201$ and 0.9215 respectively. Whereas Figure 10 shows the DPA in DMSO at $2.36 \mathrm{GHz}$ and $4.65 \mathrm{GHZ}$ with a regression of $\mathrm{R}^{2}=0.999$ and 0.828 .

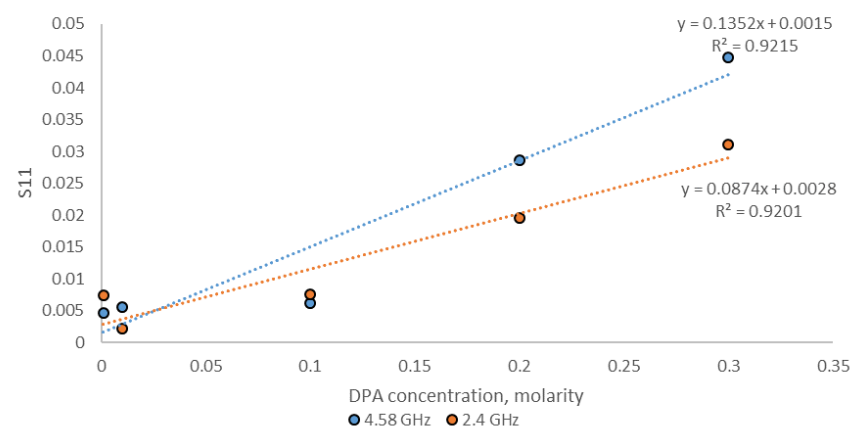

Figure 10. Trendline of $S_{11}$ vs DPA based $1 \mathrm{M} \mathrm{NaOH}$ concentration. 


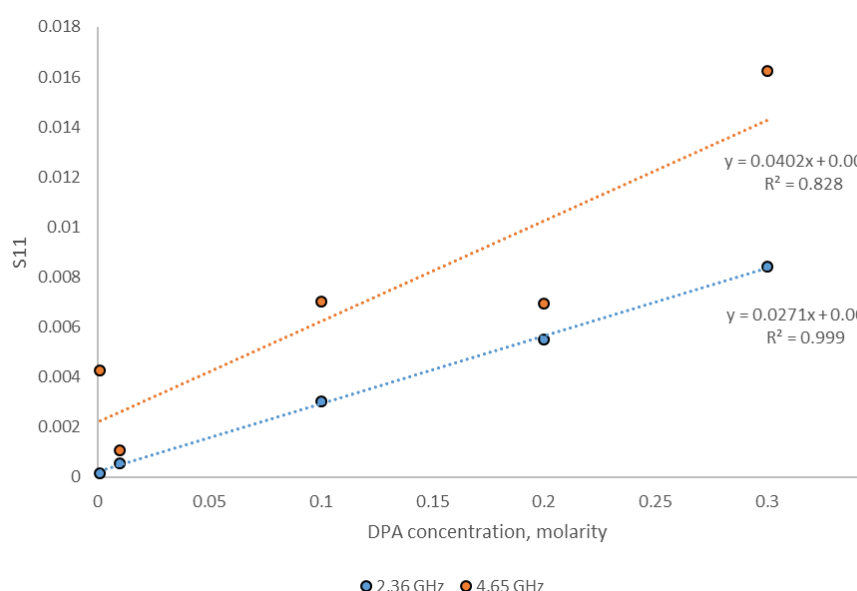

Figure 11. Trendline of $S_{11}$ vs DPA based DMSO concentration.

These findings suggest that electromagnetic wave application may have potential as a sensing instrument for detecting biological material. In future, specific horn and Vivaldi antenna will be used to direct microwave radiation to specific surfaces to examine biological material on different surfaces.

On top of that, additional experiments were conducted to examine the different species samples which consists of bacillus globigii (BG), bacillus thuringiensis (BT) media and bacillus thuringinensis (BT) spent media at constant concentration of $10^{7}$. Figure 11 shows the signal spectrum in magnitude from $2.3-2.5 \mathrm{GHz}$ for three different bacillus species. It is seen that different species yielded a different signal spectrum, which could be potential to further evaluate as a means to detection of bacillus for specially species or concentration.

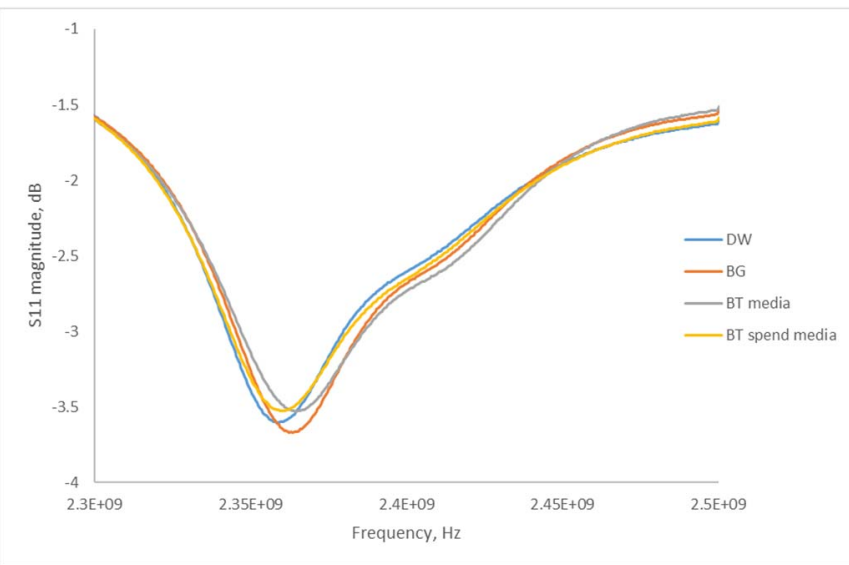

\section{Figure 12 Microwave signal spectrum for 3 different bacillus species.}

\section{CONCLUSIONS}

The EM sensor showed high potential to be used for detection of DPA in solution form, which is the bio-maker of bacillus spores. The developed $2.45 \mathrm{GHz}$ microwave cavity sensor capable to perform in non-destructive and real time application. More future works is required to investigate the use of $2.45 \mathrm{GHz}$ to detect bacillus spores on different surfaces such as concrete, wood, tiles, etc.

\section{ACKNOWLEDGMENT}

The authors gratefully acknowledge "Defence and Security Accelerator" for providing the funding for this research project under Contract DSTLX-1000131397.

\section{REFERENCES}

1. Salamon, A.W., The current world of nanomaterial characterization: discussion of analytical instruments for nanomaterial characterization. Environmental Engineering Science, 2013. 30(3): p. 101-108.

2. Saari, S.E., M.J. Putkiranta, and J. Keskinen, Fluorescence spectroscopy of atmospherically relevant bacterial and fungal spores and potential interferences. Atmospheric Environment, 2013. 71: p. 202-209.

3. Kolb, B.K., et al., Proof of bacteria and the activity of chemical and natural antibiotics by headspace gas chromatography. Journal of Analytical Science and Technology, 2019. 10(1): p. 9.

4. Ryzhov, V., Y. Hathout, and C. Fenselau, Rapid characterization of spores of Bacillus cereus group bacteria by matrix-assisted laser desorptionionization time-of-flight mass spectrometry. Applied and environmental microbiology, 2000. 66(9): p. 3828-3834.

5. Rowe-Taitt, C.A., et al., Array biosensor for detection of biohazards. Biosensors and Bioelectronics, 2000. 14(10-11): p. 785-794.

6. Sapsford, K., et al., Fluorescence - based array biosensors for detection of biohazards. Journal of applied Microbiology, 2004. 96(1): p. 47-58.

7. Tyndall, J., V. Further researches on the deportment and vital persistence of putrefactive and infective organisms from a physical point of view. Philosophical Transactions of the Royal Society of London, 1877. 167: p. 149-206.

8. Gould, G.W. and A. Hurst, The bacterial spore. 1969: London (\& New York): Academic Press. xiv $+724 \mathrm{pp}$.

9. Eski, A., Z. Demirbağ, and İ. Demir, Microencapsulation of an indigenous isolate of Bacillus thuringiensis by spray drying. Journal of microencapsulation, 2019(just-accepted): p. 1-22.

10. McCann, K. and J. Laane, Raman and infrared spectra and theoretical calculations of dipicolinic acid, dinicotinic acid, and their dianions. Journal of Molecular Structure, 2008. 890(1-3): p. 346-358.

11. Rydosz, A., et al., Microwave-based sensors with phthalocyanine films for acetone, ethanol and methanol detection. Sensors and Actuators B: Chemical, 2016. 237: p. 876-886.

12. Ateeq, M., et al., Feasibility study on using microwave sensing technique to analyse silverbased products. Journal of Electromagnetic Waves and Applications, 2016. 30(7): p. 928-944.

13. Huang, M., et al., Correlations of the impedance parameters and conductivity and permittivity of 
liquid and gel phases in a series piezoelectric quartz crystal sensor. Sensors and Actuators B: Chemical, 2001. 72(1): p. 21-27.

14. Teng, K.H., et al., Design and implementation of a non-invasive real-time microwave sensor for assessing water hardness in heat exchangers. Journal of Electromagnetic Waves and Applications, 2018. 32(7): p. 797-811. 